\title{
COVID-19 in recipients of allogeneic stem cell transplantation: favorable outcome
}

\author{
Maria Teresa Lupo-Stanghellini ${ }^{1}{ }^{1}$ - Elisabetta Xue ${ }^{1} \cdot$ Sara Mastaglio $^{1} \cdot$ Chiara Oltolini $^{2} \cdot$ Piera Angelillo $^{1}$. \\ Carlo Messina ${ }^{1}$. Simona Piemontese ${ }^{1}$ - Stefania Girlanda ${ }^{1}$ - Francesca Farina ${ }^{1}$ - Lorenzo Lazzari ${ }^{1}$. \\ Maria Pia Cicalese ${ }^{3} \cdot$ Federico Erbella $^{1} \cdot$ Raffaella Greco ${ }^{1}{ }^{1} \cdot$ Massimo Locatelli $^{4} \cdot$ Raffaella Milani $\mathbb{D}^{5}$. \\ Jacopo Peccatori ${ }^{1}$ - Consuelo Corti ${ }^{1}$ - Sarah Marktel ${ }^{1}$ - Andrea Assanelli ${ }^{1} \cdot$ Fabio Ciceri $\oplus^{1,6}$
}

Received: 12 May 2020 / Revised: 28 February 2021 / Accepted: 23 March 2021 / Published online: 30 June 2021

(c) The Author(s), under exclusive licence to Springer Nature Limited 2021

\section{To the Editor:}

The first Italian patient affected by COVID-19 was identified on 21st February 2020 and more than 3,400,000 cases have been reported in Italy so far [1]. Recently, the Italian Hematology Alliance on COVID-19 showed that patients with hematological malignancies have worse outcomes than the general population with COVID-19 and patients with hematological malignancies without COVID-19 [2].

We report the results of a prospective observational cohort study of COVID-19 in patients after allogeneic hematopoietic stem cell transplantation (HSCT) treated at the San Raffaele Scientific Institute of Milano, Italy, a tertiary care hospital located in a geographical area strongly affected by

Supplementary information The online version contains supplementary material available at https://doi.org/10.1038/s41409021-01278-x.

Fabio Ciceri

fabio.ciceri@hsr.it

1 Hematology and Bone Marrow Transplantation Unit, IRCCS San Raffaele Scientific Institute, Milano, Italy

2 Clinic of Infectious Diseases, Division of Immunology, Transplantation and Infectious Diseases, IRCCS San Raffaele Scientific Institute, Milano, Italy

3 Pediatric Immunohematology and Bone Marrow Transplantation Unit, IRCCS San Raffaele Scientific Institute, Milano, Italy

4 Laboratory Medicine Service, IRCCS San Raffaele Scientific Institute, Milano, Italy

5 Immunohematology and Transfusion Medicine Unit, IRCCS San Raffaele Scientific Institute, Milano, Italy

6 School of Medicine, Vita-Salute San Raffaele University, Milano, Italy pandemic, where 820 patients with SARS-CoV-2 infection were hospitalized between March and April 2020.

All the consecutive adult survivors who received a transplant between January 1999 and January 2020 were considered evaluable for the analysis $(n=465)$. Transplant survivors are routinely evaluated according to an allcomprehensive standardized life-time follow-up. During the time of observation (23rd Feb-27th Apr 2020), 254 patients were evaluated as previously reported [3].

A 24/7 hematologist is available by phone at our Center: any patient with concerns regarding COVID-19 was able to reach a transplant physician besides the National and Local COVID-19 emergency number.

This series is part of an institutional cohort of COVID-19 patients assessed by clinical, biological and treatment outcome data (Covid-BioB, ClinicalTrials.gov NCT04318366) at San Raffaele Scientific Institute. The study was approved by the Institutional Review Board.

According to the WHO guidance, laboratory confirmation for SARS-Cov-2 was defined as a positive result of the RT-PCR assay of nasal and pharyngeal swabs [4]. Nasopharyngeal swab test was performed according to the manufacturer's instructions. Molecular diagnosis for SARS-CoV-2 was performed through a RT-PCR qualitative assay for use on the cobas 6800/8000 Systems (Roche).

COVID-19 positive patients were classified according to clinical risk scores widely used to establish the prognosis and treatment (Supplementary Information 1).

COVID-19 patients were treated according to Institutional and National guidelines: most of the drugs (hydroxychloroquine \pm azithromycin, lopinavir/ritonavir, anakinra, colchicine, and enoxaparin) were given off-label after provision of an informed signed consent.

During the observation period, 254/465 transplanted patients were evaluated: 150 through telemedicine, 104 
via in-person visit - median time of follow-up after HSCT 5 years (r-range- 1 month-17 years). Fourteen patients were in the first 3-month period after HSCT (1/14 reported symptoms), 41 between 3 and 12 months (6/41 reported symptoms), 55 between 12 and 24 months (5/55 reported symptoms) and 144 after month 24 (12/144 reported symptoms).

The remaining patients (211) have been contacted and scheduled for a telemedicine visit.

Overall, 24/254 patients reported symptoms compatible with COVID-19: 20 patients reported fever, which was the only sign in three patients and was associated with persistent cough in 16, and/or myalgia in 3 , and/or headache in 2 . The four patients without fever reported anosmia/ageusia in one case and flu-like symptoms in 3 . Twenty-one out of the 24 symptomatic patients were tested, and three patients were not (two due to logistic difficulties, one due to patient decision). Median time from symptoms onset to nasopharyngeal swab was 3 days ( $\mathrm{r} 0-47$, three patients were tested more than 10 days after symptoms onset: 14/20/47 days), test results were available in a median time of $12 \mathrm{~h}(\mathrm{r} \mathrm{6}$ 6-24). At time of symptoms onset all the patients were requested to observe home quarantine. Median follow-up after symptoms onset was 282 days (r 53-305).

Six patients out of 254 were diagnosed with COVID-19 with positive PCR. Patients' features, ongoing treatments and major known risk factors are detailed in Table 1.

All the patients that tested negative were evaluated in-person. No one was diagnosed with influenza $\mathrm{A} / \mathrm{B}$, all presented elevated CRP and responded to empiric antibiotic therapy.

Immune reconstitution parameters (namely, recovery of Band T-cell by cytometry and immunoglobulins titres by immunoturbidimetric assays) of the six SARS-CoV-2-positive patients were evaluated during the semester before COVID-19 symptoms onset: T-cell immune-recovery was confirmed in all patients, five patients had reached a normal B-cells count -patient \#4 still suffered from absolute B cells cytopenia; recovery of total $\mathrm{IgG} / \mathrm{IgM}$ serum levels was complete in all patients, while a persistent deficit of IgA was observed in patient \#1 and patient \#4 [5]. All the patients had received anti-flu vaccination during the previous autumn.

Patient \#1 who presented mild non-respiratory symptoms and patients \#2 and \#3 with mild respiratory symptoms were all clinically stable and did not require hospitalization. They have been followed both by their general practitioner and in telemedicine by a transplant physician during the home quarantine period.

Patients \#4, \#5, and \#6 were considered clinically unstable-but not in critical conditions-and required hospitalization with respiratory support (oxygen mask).

On admission, abnormalities in chest images compatible with bilateral, interstitial pneumonia were detected (chest CT in patients \#4 and \#6, chest X-ray in patient \#5).
Risk factors identified in the general population affected by COVID-19 were analyzed in our HSCT patients: the small number of HSCT patients, however, does not allow us to draw conclusions $[6,7]$.

Data on laboratory findings and patient treatment are reported in Table 1 and Fig. 1.

All hospitalized patients received antiviral therapy (lopinavir/ritonavir) and hydroxychloroquine; patient \#5 received enoxaparin prophylaxis.

Due to persistent fever without evidence of concomitant infections, GvHD reactivation or disease relapse, additional drugs have been administered (anakinra \#5, colchicine \#4).

No patients developed acute respiratory distress syndrome (ARDS), acute cardiac injury, deep vein thrombosis and secondary infections and none required invasive management in intensive care unit.

Interestingly, patient \#6 resulted positive to pneumococcal urinary antigen at time of COVID-19 diagnosis and was treated with ceftriaxone.

The three hospitalized patients have been discharged after 37,13 , and 8 days respectively. All patients are alive and currently followed in the outpatient setting and have completely recovered from COVID-19. All the six patients had two consecutive negative swabs test: median time from the first positivity to the first negativity was 26 days ( $\mathrm{r}$ 12-70).

Four patients (\#2, \#4, \#5, and \#6) were affected by chronic GvHD (without lung involvement) at time of COVID-19 onset and 2 were receiving immunosuppressive treatment with ruxolitinib (\#4, \#6). No evidence of GvHD worsening among these patients was reported at last evaluation. Patient \#3, who had stopped ruxolitinib 6 months before -after having achieved complete resolution of chronic GvHD- experienced GvHD reactivation of mild severity.

Aggregated data of outcomes for HSCT patients infected with COVID-19 are expected soon. Meanwhile, various experiences have been reported by individual centers. Both dismal $[8-11]$ and positive $[12,13]$ outcomes were reported across transplant centers worldwide. In our center the incidence of SARS-CoV-2 symptomatic infection during the first 7 weeks of COVID-19 outbreak was $2.4 \%$. Hospitalization was necessary in $3 / 6$ patients. So far, all six patients have recovered completely and without sequelae.

Proactive search for signs and symptoms of COVID-19, prompt diagnosis and clinical management, revealed in our opinion crucial towards a positive outcome.

In our limited experience, the chance of cure from COVID-19 in the frail population of HSCT recipients was superimposable to the general population: it should be emphasized that this observation is based on a limited number of cases in a single center. The evaluation of aggregated data is essential to define the prognosis and the 
Table 1 Patients features and risk factors/laboratory values associated with mortality.

\begin{tabular}{|c|c|c|c|c|c|c|}
\hline & $\mathrm{Pt} \# 1$ & $\mathrm{Pt} \# 2$ & $\mathrm{Pt} \# 3$ & $\mathrm{Pt} \# 4$ & $\mathrm{Pt} \# 5$ & $\mathrm{Pt} \# 6$ \\
\hline Sex & M & M & M & M & $\mathrm{F}$ & $\mathrm{F}$ \\
\hline Age & 28 & 46 & 60 & 55 & 74 & 34 \\
\hline Diagnosis & AML & AML & AML & AML & MDS & AML \\
\hline Donor & MMRD & MUD & MUD & MMRD & MUD & MUD \\
\hline Time post HSCT & $12 \mathrm{~m}$ & $43 \mathrm{~m}$ & $36 \mathrm{~m}$ & $40 \mathrm{~m}$ & $8 \mathrm{y}$ & $14 \mathrm{y}$ \\
\hline $\mathrm{T} / \mathrm{B}$-cell recovery & $+/+$ & $+/+$ & $+/+$ & $+l-$ & $+/+$ & $+/+$ \\
\hline $\mathrm{IgG} / \operatorname{Ig} \mathrm{A} / \mathrm{IgM}$ recovery & $+/-I+$ & $+/+/+$ & $+/+/+$ & $+/-/+$ & $+/+/+$ & $+/+/+$ \\
\hline Chronic GvHD & No & Yes & Yes & Yes & Yes & Yes \\
\hline $\begin{array}{l}\text { Chronic GvHD severity and } \\
\text { site at COVID-19 onset }\end{array}$ & n.a. & $\begin{array}{l}\text { Severe } \\
\text { Joint and Fascia }\end{array}$ & Resolved & $\begin{array}{l}\text { Mild } \\
\text { Skin }\end{array}$ & $\begin{array}{l}\text { Mild } \\
\text { Eyes }\end{array}$ & $\begin{array}{l}\text { Severe } \\
\text { Skin, Mouth, Eyes, Joint } \\
\text { and Fascia }\end{array}$ \\
\hline Systemic IST & No & Imatinib/MTX & No & Ruxolitinib & No & Ruxolitinib \\
\hline Symptoms & Anosmia Ageusia & $\begin{array}{l}\text { Fever } \\
\text { Headache }\end{array}$ & $\begin{array}{l}\text { Fever } \\
\text { Cough }\end{array}$ & $\begin{array}{l}\text { Fever } \\
\text { Cough }\end{array}$ & $\begin{array}{l}\text { Fever } \\
\text { Cough }\end{array}$ & $\begin{array}{l}\text { Fever } \\
\text { Myalgia }\end{array}$ \\
\hline MEWS & 0 & 2 & 2 & 3 & 5 & 3 \\
\hline PPSv2 & $100 \%$ & $80 \%$ & $80 \%$ & $40 \%$ & $40 \%$ & $50 \%$ \\
\hline CIRS & 4 & 9 & 6 & 13 & 10 & 15 \\
\hline PSI & n.a. & n.a. & n.a. & III & IV & III \\
\hline APACHE & n.a. & n.a. & n.a. & 13 & 16 & 11 \\
\hline $\begin{array}{l}\text { Outcome } \\
\qquad\left(\mathrm{n}^{*} ; \mathrm{n}^{* *}\right)\end{array}$ & $\begin{array}{l}\text { Resolved } \\
(280 ; \text { n.a. })\end{array}$ & $\begin{array}{l}\text { Resolved } \\
(305 ; \text { n.a. })\end{array}$ & $\begin{array}{l}\text { Resolved } \\
(285 ; \text { n.a. })\end{array}$ & $\begin{array}{l}\text { Discharged } \\
\text { Resolved } \\
(286 ; 37)\end{array}$ & $\begin{array}{l}\text { Discharged } \\
\text { Resolved } \\
(278 ; 13)\end{array}$ & $\begin{array}{l}\text { Discharged } \\
\text { Resolved } \\
(291 ; 8)\end{array}$ \\
\hline \multicolumn{7}{|l|}{ Risk factors } \\
\hline Age $\geq 60 y$ & No & No & Yes & No & Yes & No \\
\hline Male gender & Yes & Yes & Yes & Yes & No & No \\
\hline Hypertension & No & No & Yes & Yes & No & Yes \\
\hline Cardiovascular disease & No & No & No & Yes & No & No \\
\hline Diabetes & No & No & No & No & No & No \\
\hline Chronic lung disease & No & Yes & No & No & Yes & No \\
\hline \multicolumn{7}{|l|}{ Laboratory value } \\
\hline Elevated LDH (>220 U/L) & n.a. & n.a. & n.a. & 404 & 303 & 322 \\
\hline Elevated CRP (>6 mg/L) & n.a. & n.a. & n.a. & 153 & 248 & 99 \\
\hline Elevated D-dimer $(>0.5 \mathrm{mcg} / \mathrm{L})$ & n.a. & n.a. & n.a. & No & 1.6 & No \\
\hline Elevated PT inr (ratio > 1.18) & n.a. & n.a. & n.a. & No & 0.93 & No \\
\hline Platelets $<100 \times 10^{9} / \mathrm{L}$ & n.a. & n.a. & n.a. & 209 & 141 & 266 \\
\hline Lymphocytes $<0.8 \times 10^{9} / \mathrm{L}$ & n.a. & n.a. & n.a. & 0.4 & 1.9 & 0.9 \\
\hline Ground glass opacities & n.a. & n.a. & n.a. & Yes & No & Yes \\
\hline Bilateral pneumonia & n.a. & n.a. & n.a. & Yes & Yes & Yes \\
\hline $\mathrm{PaO}_{2} / \mathrm{FiO}_{2}<300 \mathrm{mmHg}$ & n.a. & n.a. & n.a. & No & Yes & No \\
\hline
\end{tabular}

$A M L$ acute myeloid leukemia, $M D S$ myelodysplastic syndrome, $M M R D$ mismatch related donor, $M U D$ match unrelated donor, $H S C T$ allogeneic hematopoietic stem cell transplantation, GvHD Graft-versus-Host-Disease, IST immunosuppressive treatment, MEWS modified early warning score, $P P S v 2$ palliative performance scale, CIRS cumulative illness rating scale, $P S I$ pneumonia severity index, APACHE II acute physiology and chronic health evaluation II, $m$ months, MTX methotrexate, $C R P$ C-reactive protein, $L D H$ lactate dehydrogenase, $P T$ inr prothrombin time international normalized ratio, $\mathrm{Pao}_{2}$ arterial oxygen partial pressure, $\mathrm{Fio}_{2}$ fractional inspired oxygen, $y$ years, $n *$ days since symptoms presentation, $n * *$ days of hospitalization, n.a. not applicable, n.d. not done.

possibility of treatment in this setting. Patients with mild symptoms did not require hospitalization and were safely managed under strict surveillance at home. Exploitation of telemedicine was crucial.
In our experience, ongoing immunosuppressive therapy did not seem to affect the outcome.

Overall, we cannot draw any conclusions regarding the efficacy of all antiviral/immunomodulatory therapies 


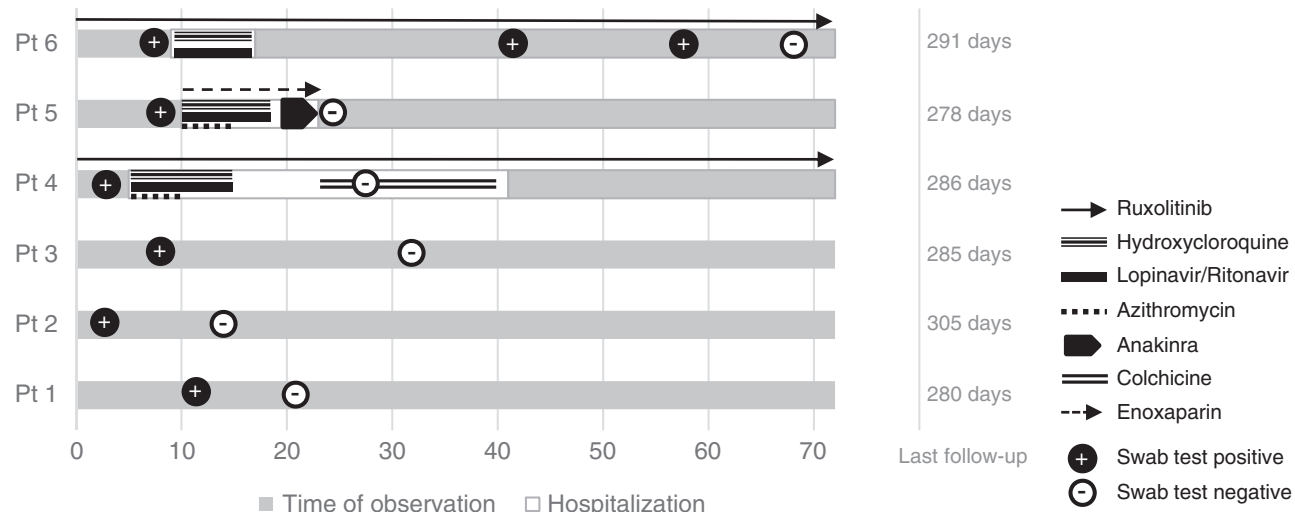

Fig. 1 Patients' distribution over time and treatment. Patient 1-3 did not receive a specific COVID-19 treatment. Ruxolitinib dosage: $5 \mathrm{mg}$ q12h; hydroxychloroquine dosage: $200 \mathrm{mg}$ q12h; lopinavir/ritonavir

administered to our patients so far; nevertheless, we can assert that no drug-related toxicities were reported in our cohort.

In conclusion, being a recipient of HSCT should not be considered a priori a determinant of dismal prognosis at diagnosis of COVID-19.

Author contributions MTLS and FC designed the study. MTLS, EX, SM, CO, SP, SG, FF, LL, and AA collected and summarized the epidemiological and clinical data; participated in the performance of the research. MTLS, EX, SM, CO, CM, SP, SG, FF, LL, MPC, FE, RG, ML, RM, JP, CC, SM, AA, and FC drafted the paper. MTLS and FC revised the final paper.

\section{Compliance with ethical standards}

Conflict of interest The author declares no competing interests.

Publisher's note Springer Nature remains neutral with regard to jurisdictional claims in published maps and institutional affiliations.

\section{References}

1. Italian Ministry of Heatlh official web-site https://opendatadpc.maps. arcgis.com/apps/dashboards/b0c68bce2cce478eaac82fe38d4138b1. Accessed 23 Mar 2021.

2. Passamonti F, Cattaneo C, Arcaini L, Bruna R, Cavo M, Merli F, et al. Clinical characteristics and risk factors associated with COVID-19 severity in patients with haematological malignancies in Italy: a retrospective, multicentre, cohort study. Lancet Haematol. 2020;7:e737-45. https://doi.org/10.1016/S2352-3026(20) 30251-9.

3. Lupo-Stanghellini MT, Messina C, Marktel S, Carrabba MG, Peccatori J, Corti C, et al. Following-up allogeneic transplantation recipients during the COVID-19 pandemic. Lancet Haematol. 2020;7:e564-5. https://doi.org/10.1016/S2352-3026(20)30176-9. dosage: 400/100 mg q12h; azithromycin dosage: $500 \mathrm{mg}$ day $1,250 \mathrm{mg}$ daily subsequently; anakinra dosage: $5 \mathrm{mg} / \mathrm{Kg}$ q12h; colchicine dosage: $1 \mathrm{mg}$ daily; enoxaparin dosage: $4000 \mathrm{UI}$ q12h.

4. WHO. Clinical management of severe acute respiratory infection when novel coronavirus ( $\mathrm{nCoV})$ infection is suspected: interim guidance, 25 January 2020. Published January 25, 2020. https://a pps.who.int/iris/handle/10665/330854. Accessed 30 Mar 2020.

5. van den Brink MR, Velardi E, Perales MA. Immune reconstitution following stem cell transplantation. Hematol Am Soc Hematol Educ Progr. 2015;2015:215-9.

6. Grasselli G, Zangrillo A, Zanella A, Antonelli M, Cabrini L, Castelli A, et al. Baseline characteristics and outcomes of 1591 patients infected with SARS-CoV-2 admitted to ICUs of the Lombardy Region, Italy. JAMA. 2020;323:1574-81. https://doi. org/10.1001/jama.2020.5394.

7. Ciceri F, Castagna A, Rovere-Querini P, De Cobelli F, Ruggeri A, Galli L, et al. Early predictors of clinical outcomes of COVID-19 outbreak in Milan, Italy. Clin Immunol. 2020;217:108509. https:// doi.org/10.1016/j.clim.2020.108509.

8. Huang J, Lin H, Wu Y, Fang Y, Kumar R, Chen L, et al. COVID19 in posttransplant patients-report of 2 cases. Am J Transplant. 2020;20:1879-81. https://doi.org/10.1111/ajt.15896.

9. He W, Chen L, Chen L, Yuan G, Fang Y, Chen W, et al. COVID19 in persons with haematological cancers. Leukemia. 2020;34:1637-45. https://doi.org/10.1038/s41375-020-0836-7.

10. Shah V, Ko Ko T, Zuckerman M, Vidler J, Sharif S, Mehra V, et al. Poor outcome and prolonged persistence of SARS-CoV-2 RNA in COVID-19 patients with haematological malignancies; King's College Hospital experience. Br J Haematol. 2020;190: e279-82. https://doi.org/10.1111/bjh.16935.

11. Martín-Moro F, Marquet J, Piris M, Michael BM, Sáez AJ, Corona $\mathrm{M}$, et al. Survival study of hospitalised patients with concurrent COVID-19 and haematological malignancies. $\mathrm{Br} \mathrm{J}$ Haematol. 2020;190:e16-20. https://doi.org/10.1111/bjh.16801.

12. Malard F, Genthon A, Brissot E, van de Wyngaert Z, Marjanovic Z, Ikhlef S, et al. COVID-19 outcomes in patients with hematologic disease. Bone Marrow Transplant. 2020;55:2180-4. https:// doi.org/10.1038/s41409-020-0931-4.

13. Shah GL, DeWolf S, Lee YJ, Tamari R, Dahi PB, Lavery JA, et al. Favorable outcomes of COVID-19 in recipients of hematopoietic cell transplantation. J Clin Investig. 2020;130:6656-67. https://doi.org/10.1172/JCI141777. 